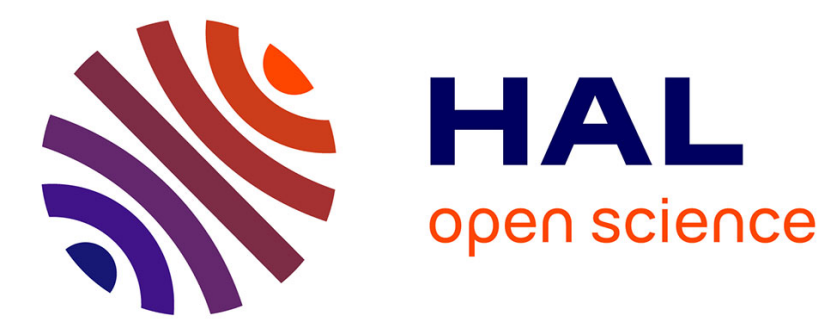

\title{
ABSORPTION ET PRODUCTION DE MÉSONS П
}

P.Y. Bertin

\section{To cite this version:}

P.Y. Bertin. ABSORPTION ET PRODUCTION DE MÉSONS П. Journal de Physique Colloques, 1975, 36 (C5), pp.C5-13-C5-20. 10.1051/jphyscol:1975503 . jpa-00216356

\section{HAL Id: jpa-00216356 https://hal.science/jpa-00216356}

Submitted on 1 Jan 1975

HAL is a multi-disciplinary open access archive for the deposit and dissemination of scientific research documents, whether they are published or not. The documents may come from teaching and research institutions in France or abroad, or from public or private research centers.
L'archive ouverte pluridisciplinaire HAL, est destinée au dépôt et à la diffusion de documents scientifiques de niveau recherche, publiés ou non, émanant des établissements d'enseignement et de recherche français ou étrangers, des laboratoires publics ou privés. 


\title{
ABSORPTION ET PRODUCTION DE MÉSONS $\Pi$
}

\author{
P. Y. BERTIN
}

Laboratoire de Physique Corpusculaire, Université de Clermont, B.P. 45, 63170 Aubière, France

\begin{abstract}
Résumé. - Les expériences d'émission et d'absorption en vol et au repos de mésons $I$, réalisées récemment en Europe, sont présentées. Il est montré que dans la compréhension des résultats peuvent intervenir des mécanismes plus complexes que ceux à un nucléon et qu'un travail important et excitant sur le plan expérimental reste à réaliser.
\end{abstract}

Abstract. - The experiments about pion production and pion absorption at rest or in fly recently performed in Europ are presented. It is shown that to understand the data some reaction's mechanism more complex than the one nucleon mechanism must be introduced and it is concluded that important and exciting experimental work is still necessary.

Depuis plusieurs années, nous voyons apparaître des machines spécialisées, appelées Usines à Pion, accélérateurs d'intensité prévue importante, allant jusqu'à 100 , voire $1000 \mu \mathrm{A}$ moyens de protons d'énergie relativement basse, de 500 à $800 \mathrm{MeV}$. Le but principal de tels appareils est la production de mésons $\Pi$ en nombre considérable $\left(10^{6}\right.$ à $\left.10^{10} \Pi / \mathrm{s}\right)$ de basse énergie ( 0 à $400 \mathrm{MeV}$ ).

L'effort entrepris dans cette voie est apparu nécessaire du fait même des mésons $\Pi$ en tant que sonde de la matière nucléaire.

Rappelons que le méson est tout d'abord un boson, et qu'il obéit à l'équation de Klein-Gordon,

- que son spin isotopique est unité, et qu'il se présente donc sous trois états de charge,

- que sa masse, $139 \mathrm{MeV}$, est bien plus élevée que celle de l'électron tout en restant d'un ordre de grandeur inférieur à celle des nucléons,

- que la portée des forces nucléaires (longueur de diffusion pion-nucléon $\simeq 1 \times 10^{-13} \mathrm{~cm}$ ) est petite, et que lorsque l'énergie des pions n'est pas très élevée, seuls les moments angulaires d'ordre faible (ondes $\mathbf{S}$ et $\mathrm{P})$ seront impliqués,

- que sa durée de vie, au moins pour les états de charge non nuls, $2,6 \times 10^{-8} \mathrm{~s}$, est suffisamment grande pour ne pas impliquer trop de difficultés expérimentales,

- et enfin qu'à ce jour, dans le domaine d'énergie qui nous intéresse, les modes de production à partir des nucléons $(\gamma \mathrm{N} \rightarrow \mathrm{N}+\Pi, \mathrm{N}+\mathrm{N} \rightarrow \mathrm{N}+\mathrm{N}+\Pi)$ ou ses interactions avec ceux-ci $(\Pi \mathrm{N} \rightarrow \Pi \mathrm{N})$ sont connus avec une précision qui, si elle n'est pas parfaite, est largement suffisante pour être utilisée en physique nucléaire. Nous donnons à titre d'exemple les sections efficaces différentielles obtenues aux énergies inférieures à $100 \mathrm{MeV}$ pour la réaction $\Pi^{+} \mathrm{p} \rightarrow \Pi^{+} \mathrm{p}$ (Fig. 1 et 2) [1] et les résultats de la réaction $\Pi^{+} \mathrm{d} \rightarrow \mathrm{pp}$ (Fig. 3) [2] obtenus respectivement à Saclay et à Los Alamos.

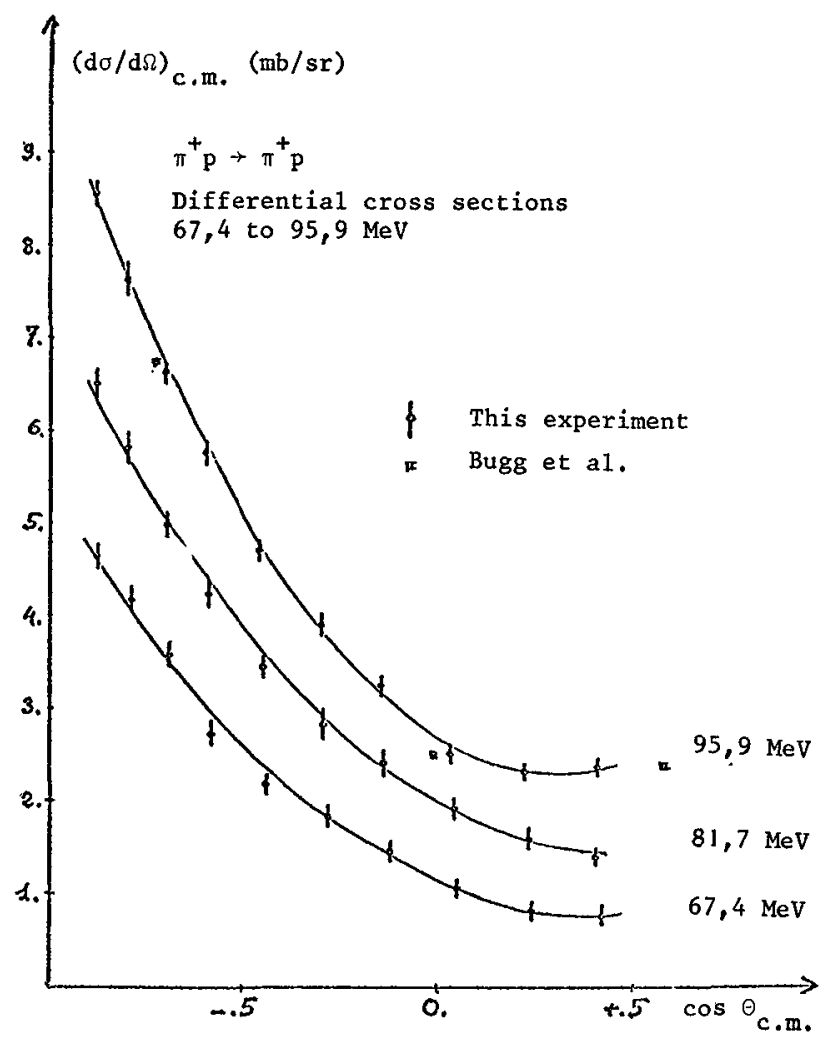

FIG. 1. - Section efficace différentielle de la réaction $\Pi^{+} \mathrm{p} \rightarrow \Pi^{+} \mathrm{p}$ en fonction de l'angle dans le centre de masse, pour des énergies $T_{I I}=67,4 \mathrm{MeV}, 81,7 \mathrm{MeV}, 95,9 \mathrm{MeV}$. 


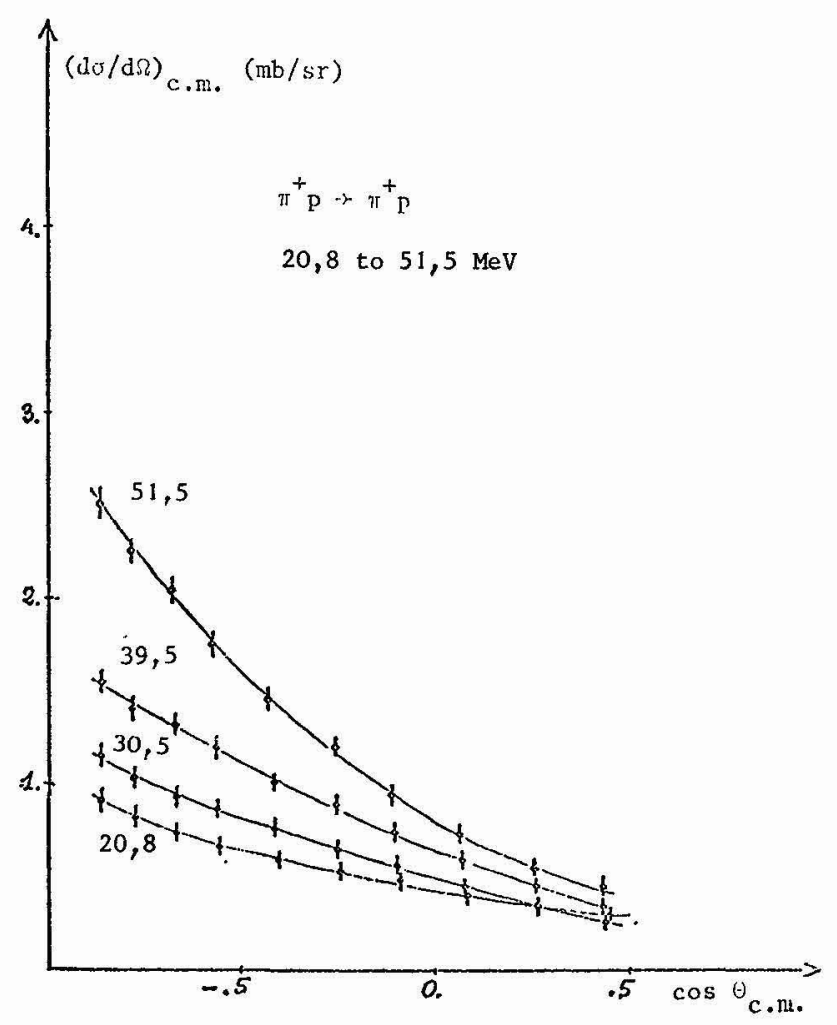

FIG. 2. - Idem, mais avec $T_{I I}=51,5 \mathrm{MeV}, 39,5 \mathrm{MeV}, 30,5 \mathrm{MeV}$, $20,8 \mathrm{MeV}$.

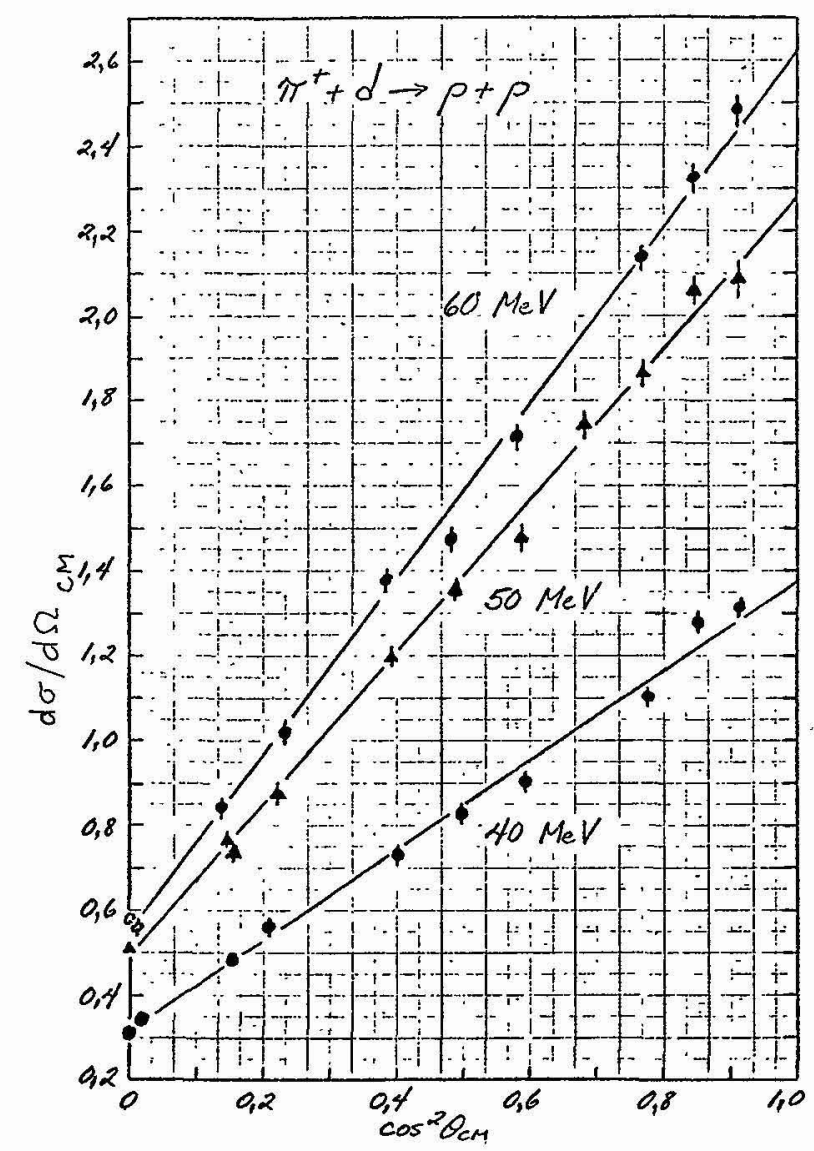

Fig. 3. - Section efficace différentielle de la réaction $\Pi^{+} \mathrm{d} \rightarrow \mathrm{pp}$ en fonction de $\cos ^{2} \theta_{\mathrm{cm}}$ pour $T_{\Pi}=60,50,40 \mathrm{MeV}$.
Dans l'immense domaine de physique nucléaire ouvert par les Usines à Pion, nous nous attacherons plus spécialement aux réactions où le pion disparaît, c'est-à-dire les réactions d'absorption. Mais dans un premier temps, je voudrais essayer de préciser les problèmes expérimentaux que pose ce type de réaction.

Le Pion, en tant que boson, peut être absorbé par le noyau, c'est-à-dire disparaître dans la réaction; il laisse donc au noyau une quantité importante d'énergie, $139 \mathrm{MeV}$ au moins. Le système ayant absorbé cette énergie peut se désexciter par un processus d'émission de plusieurs nucléons ou d'amas de nucléons. C'est alors un phénomène d'évaporation, remarquons que nous employons ce terme dans un sens très large, $\mathrm{y}$ incorporant aussi l'émission de nucléons résultants de la présence d'état non lié des noyaux. Mais lorsqu'on étudie l'absorption de pions, on s'aperçoit que quelques nucléons ou noyaux légers sont émis à grande énergie, ce qui nous conduit à envisager un processus en deux étapes. L'absorption proprement dite sur une partie du noyau correspond à ces fragments de haute énergie, suivie d'une désexcitation du noyau résiduel par évaporation. Les renseignements sur la structure nucléaire que l'on peut espérer tirer de telle étude résident en fait dans la première phase de la réaction.

Un tel schéma implique du point de vue expérimental plusieurs commentaires, et en particulier :

- Il est impossible d'espérer séparer les deux étapes du processus dans le temps.

- Le mécanisme direct est associé avec les particules les plus énergétiques, mais son espace de phase autorise aussi des énergies analogues à celle de l'évaporation.

Il en résulte que nous ne pourrons étudier que certains cas précis, bien particuliers, par exemple :

- Le cas où l'énergie d'excitation laissée au noyau est suffisamment faible pour que celui-ci reste dans un état lié, et ceci sera d'autant mieux réalisé que l'on aura affaire à des noyaux légers et près de la ligne de stabilité.

- Les réactions inverses de l'absorption : les productions de pions.

Remarquons que pour obtenir une information sur les propriétés de la matière nucléaire, il est indispensable de se placer dans des conditions expérimentales telles que le processus considéré ne soit pas dominé par l'interaction pion-nucléon. Il faudra donc choisir des énergies de pions extérieures à la bande $100-400 \mathrm{MeV}$ qui correspond à la résonance $\left(\frac{3}{2} \frac{3}{2}\right)$.

Il m'est facile maintenant de présenter les contributions que j'ai à rapporter, puisqu'elles vont illustrer ce que nous venons de voir.

1. Absorption de $\Pi^{+}$en vol. - Présentons tout d'abord les travaux de Bachelier et al. qui étudient la réaction ${ }^{16} \mathrm{O}\left(\Pi^{+}, p\right){ }^{15} \mathrm{O}^{*}$ à $70 \mathrm{MeV}$. Cette réaction 
est à rapprocher de celle réalisée par J. Amato et al. [3] à la même énergie, et à un angle d'émission du proton de $25^{\circ}$ sur ${ }^{16} \mathrm{O}$. Je présente ce résultat (Fig. 4) pour indiquer les difficultés expérimentales de ce type d'expérience. Notons au passage l'ordre de grandeur des sections efficaces intégrées de 0 à $8 \mathrm{MeV}$ : $15 \pm 8 \mu \mathrm{b} / \mathrm{Sr}$ ! De même, la figure 5 montre des résultats relatifs à ${ }^{12} \mathrm{C}$ obtenus à un angle de $11^{\circ}$ par T. R. Witten et al. [4]. Dans ces deux cas, la résolution expérimentale ne permet pas de voir les états excités.

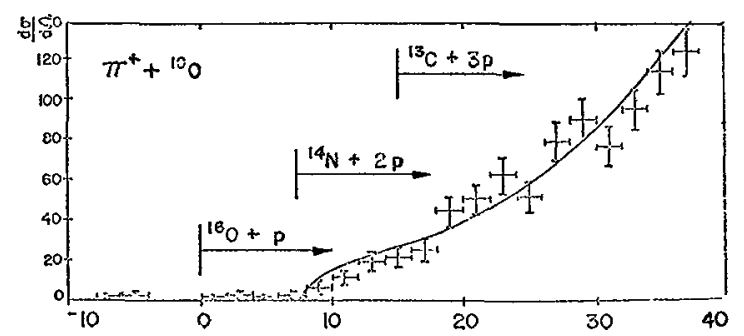

Fig. 4. - Excitation du noyau résiduel lors de la réaction $\Pi^{+}+{ }^{16} \mathrm{O}$ à $T_{\Pi}=70 \mathrm{MeV}$, le proton étant détecté à $25^{\circ}$.

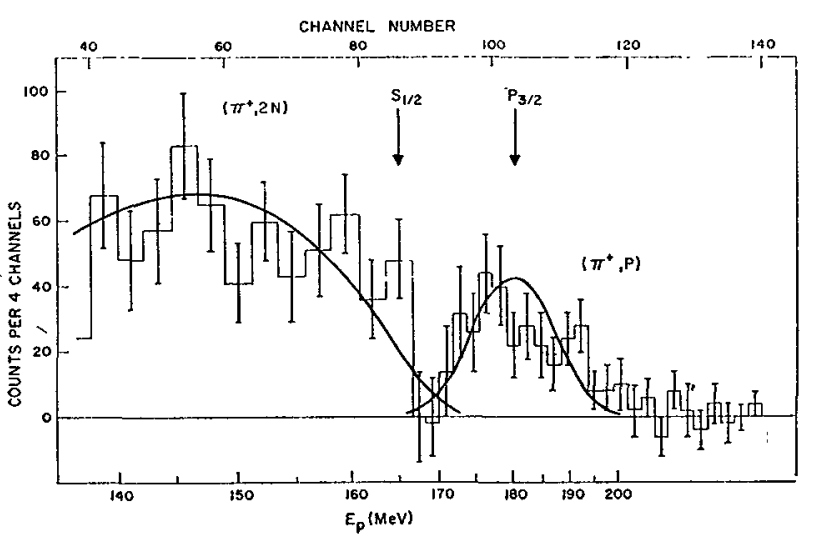

Fig. 5. - Energie du proton lors de la réaction $\Pi^{+}+{ }^{12} \mathrm{C} \rightarrow \mathrm{p}+\mathrm{X}$, le proton étant détecté à $11^{\circ}$.

Avec l'expérience réalisée par le groupe de l'I.P.N., c'est une véritable distribution angulaire qui a été obtenue pour plusieurs états de ${ }^{15} \mathrm{O}$, états $\frac{1}{2}^{-}$et et $\frac{3}{2}^{-}(6,18 \mathrm{MeV})$. La résolution obtenue avec un télescope à parcours, comportant 13 plastiques scintillants et un absorbeur de carbone d'épaisseur variable, est de 3,2 MeV. L'énergie choisie est comparable aux expériences plus anciennes, et de plus correspond à une énergie maximum de protons de $185 \mathrm{MeV}$. Cette énergie est comparable à celle des protons incidents utilisés dans un autre type d'expérience sur lequel nous reviendrons par la suite.

Dans ce type d'expérience, le mécanisme le plus simple que l'on peut imaginer est un mécanisme à un nucléon de type Pick-up, analogue à celui intervenant dans les réactions $(\mathrm{p}, \mathrm{d})$ ou $\left({ }^{3} \mathrm{He}, \alpha\right)[5,6]$, à des énergies de $155 \mathrm{MeV}$ pour les protons et de $216 \mathrm{MeV}$ pour les ${ }^{3} \mathrm{He}$ (Fig. 6 et 7). Nous voyons que pour ces. deux réactions la production des états $\frac{3}{2}^{-}$et $\frac{1}{2}^{-}$se

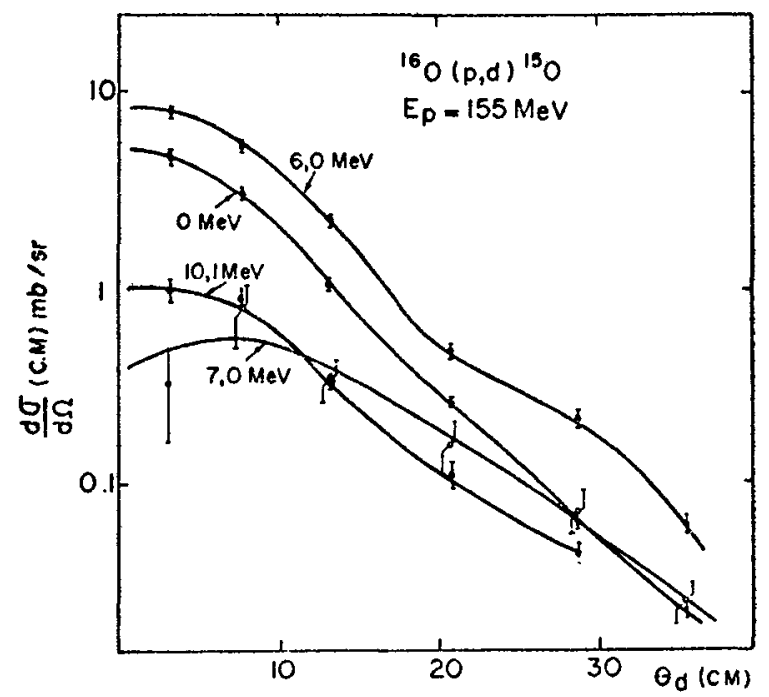

FIG. 6. - Distribution angulaire de la réaction ${ }^{16} \mathrm{O}(\mathrm{p}, \mathrm{d})^{15} \mathrm{O}$ pour les états $\frac{1}{2}^{-}$et $\frac{3}{2}^{-}, E_{\mathbf{p}}=155 \mathrm{MeV}$.

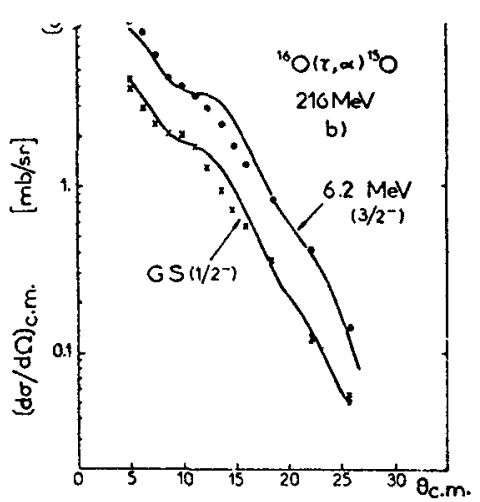

FIG. 7. - Distribution angulaire de la réaction ${ }^{16} \mathrm{O}\left({ }^{3} \mathrm{He}, \alpha\right){ }^{15} \mathrm{O}$, pour les états $\frac{1}{2}^{-}$et $\frac{3}{2}^{-}$.

fait dans un rapport de 2 quel que soit l'angle d'émission.

Avec les $\Pi^{+}$, D. Bachelier et al. observent un rapport de 10 (Fig. 9). Naïvement, on peut remarquer que les $\Pi^{+}$disparaissant dans la réaction il y a plus d'énergie disponible que pour les réactions $(\mathrm{p}, \mathrm{d})$ et $\left({ }^{3} \mathrm{He}, \alpha\right)$, et qu'alors l'espace de phase doit être différent. Ce rapport de production de 10 , et le fait que l'on observe aussi le niveau $T=\frac{3}{2}$ (Fig. 8) de ${ }^{15} \mathrm{O}$ qui ne peut pas être excité par un mécanisme à un nucléon, conduisent les auteurs à proposer un mécanisme à deux nucléons.

2. Réaction de production. - Le deuxièmé type de réaction que je veux exposer est celui des réactions inverses de capture, c'est-à-dire les réactions de production de pion au voisinage du seuil.

Celles-ci sont caractérisées par de très grands transferts de moment $\left(2,5 \mathrm{fm}^{-1}\right)$, présentant une faible variation avec l'angle d'émission du pion. Le travail que je veux présenter ici est une extension des très belles expériences réalisées à Uppsala par $\mathrm{S}$. Dahlgren et al. $[7,8]$ à partir de protons de $185 \mathrm{MeV}$ (à rappro- 


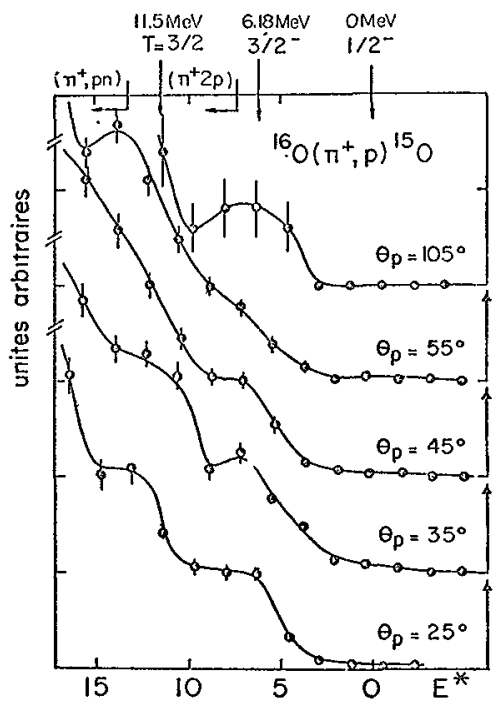

Fig. 8. - Spectre d'excitation de ${ }^{15} \mathrm{O}$ dans la réaction ${ }^{16} \mathrm{O}\left(\Pi^{+}, \mathrm{p}\right){ }^{15} \mathrm{O}$ pour $T_{n u}=70 \mathrm{MeV}$ et les angles d'émission du proton de $25^{\circ}, 35^{\circ}$, $45^{\circ}, 55^{\circ}$ et $105^{\circ}$

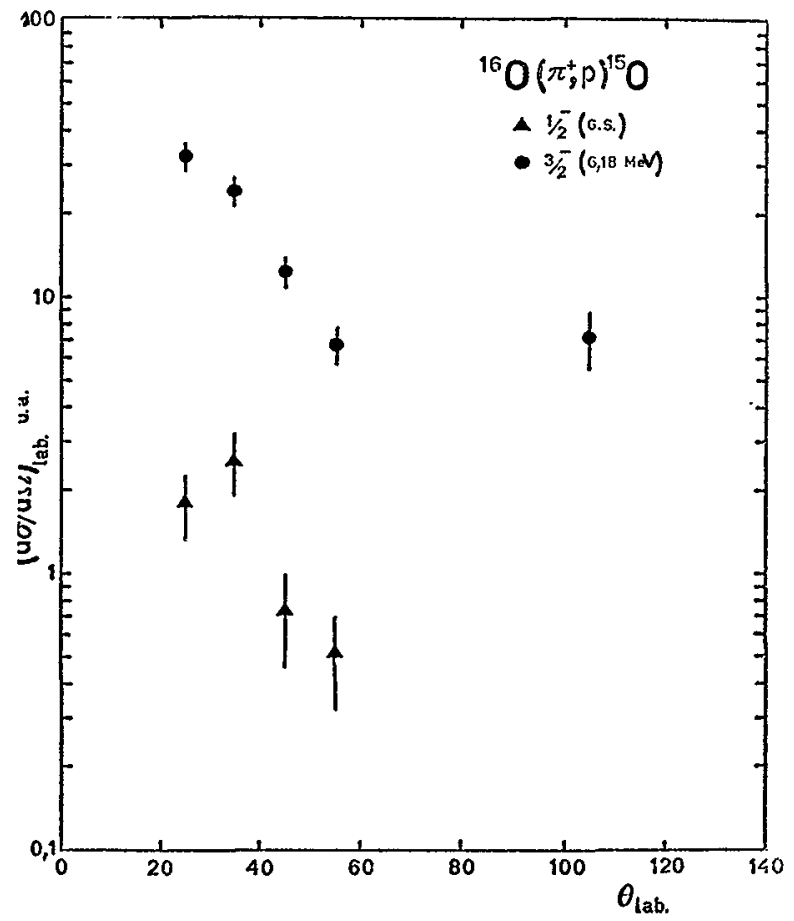

Fig. 9. - Distribution angulaire de la réaction ${ }^{16} \mathrm{O}\left(\Pi^{+}, \mathrm{p}\right)^{15} \mathrm{O}$ pour le grand stat et l'état 6,18 $\mathrm{MeV}$ de ${ }^{15} \mathrm{O}$.

cher de l'énergie maximum des protons émis dans l'expérience de D. Bachelier) sur des cibles de ${ }^{12} \mathrm{C}$, ${ }^{9} \mathrm{Be}$ et ${ }^{40} \mathrm{Ca}$... La figure 10 représente l'énergie d'excitation de la réaction ${ }^{10} \mathrm{Be}\left(\mathrm{p}, \Pi^{+}\right){ }^{11} \mathrm{Be}$. Y. Le Bornec et al. [9, 10], à l'I.P.N. d'Orsay, ont étendu ces mesures vers les basses énergies, $154 \mathrm{MeV}$ pour ${ }^{10} \mathrm{Be}$ (Fig. 11), et ont étudié la variation de la section efficace ${ }^{40} \mathrm{Ca}\left(\mathrm{p}, \Pi^{+}\right){ }^{41} \mathrm{Ca}_{\mathrm{gs}}$ à transfert constant en fonction de l'énergie (Fig. 12 et 13). Leur dispositif expérimental (Fig. 14) esst un spectromètre magnétique.
Le résultat le plus intéressant, dont les auteurs ne peuvent tirer de conclusion, leur interprétation n'étant pas assez avancée, est que cette section efficace à transfert constant décroit d'un facteur 7,4 entre 185 et $154 \mathrm{MeV}$, et d'un facteur 7 entre 154 et $149 \mathrm{MeV}$, alors que l'espace de phase conduit à des facteurs 5 et 4,3 respectivement.

Signalons la complexité des calculs, en rappelant les diagrammes des deux mécanismes les plus souvent utilisés pour interpréter ce genre de réaction : le mécanisme à un nucléon (Fig. 15) et le mécanisme à deux nucléons (Fig. 16).

Notons qu'en plus des ingrédients nécessaires aux mécanismes proprement dits (par exemple, pour le mécanisme à deux nucléons, les résultats relatifs à la réaction $\mathrm{N}+\mathrm{N} \rightarrow \mathrm{NN}+\Pi$, ou tout autre calcul plus fondamental), il faut tenir compte de la distorsion de la fonction d'onde du proton incident et de l'interaction dans l'état final.

Z. Grossman, F. Lenz et M. P. Locher ont effectué un calcul très complet pour les expériences d'Uppsala relatives au ${ }^{12} \mathrm{C}$ [11]. Ils montrent que le processus doit être dominé par un mécanisme à deux nucléons avec formation d'un $\mathrm{N}^{*}$ virtuel. M. Dilling, H. M. Hofman et M. G. Hubert [12] utilisent uniquement un mécanisme à un nucléon, en introduisant des corrélations à courte portée entre deux nucléons, tant dans l'état initial que dans l'état final; les résultats qu'ils obtiennent sont aussi très satisfaisants, mais nous pouvons nous poser la question de savoir si l'introduction de corrélations à courte portée ne revient pas à prendre en compte d'une autre manière le processus à deux nucléons.

3. Capture de $\Pi^{-}$à l'arrêt. - Je veux signaler ici les expériences réalisées par $\mathrm{B}$. Coupat et al. à l'A.L.S. [13] sur la réaction $\Pi^{-}+{ }^{12} \mathrm{C} \rightarrow{ }^{11} \mathrm{Be}+\mathrm{p}$ avec des pions à l'arrêt par une méthode d'activation. Le résultat, à savoir la probabilité d'une telle réaction intégrée sur le fondamental et le premier état du ${ }^{11} \mathrm{Be}$ (seul état lié du ${ }^{11} \mathrm{Be}$ ), est de $5 \times 10^{-4}$ par pion absorbé, chiffre relativement élevé. Le mécanisme est a fortiori plus complexe que dans les autres réactions, puisque pour obtenir l'émission d'un proton à partir d'un $\Pi^{-}$, il est indispensable d'envisager un mécanisme plus séquentiel. Signalons aussi pour ce même groupe [14] le résultat similaire obtenu sur ${ }^{14} \mathrm{~N}$, mais dans ce cas la technique d'activation a été remplacée par une mesure de l'énergie du proton émis (Fig. 17). Ce type d'expérience, à ce stade, est difficile à interpréter, car le résultat est un nombre unique, et ne permet donc de fixer qu'un seul paramètre des mécanismes envisagés. Mais ces expériences se veulent avant tout exploratoires, et par là sont très intéressantes.

Enfin, je terminerai la revue des expériences que j'ai à rapporter par celle de H. D. Engelhardt, qui étudie non plus l'absorption de $\Pi^{-}$à l'arrêt par détection des particules rapides, mais par spectrométrie $\gamma$ du noyau résiduel $[15,16]$. 


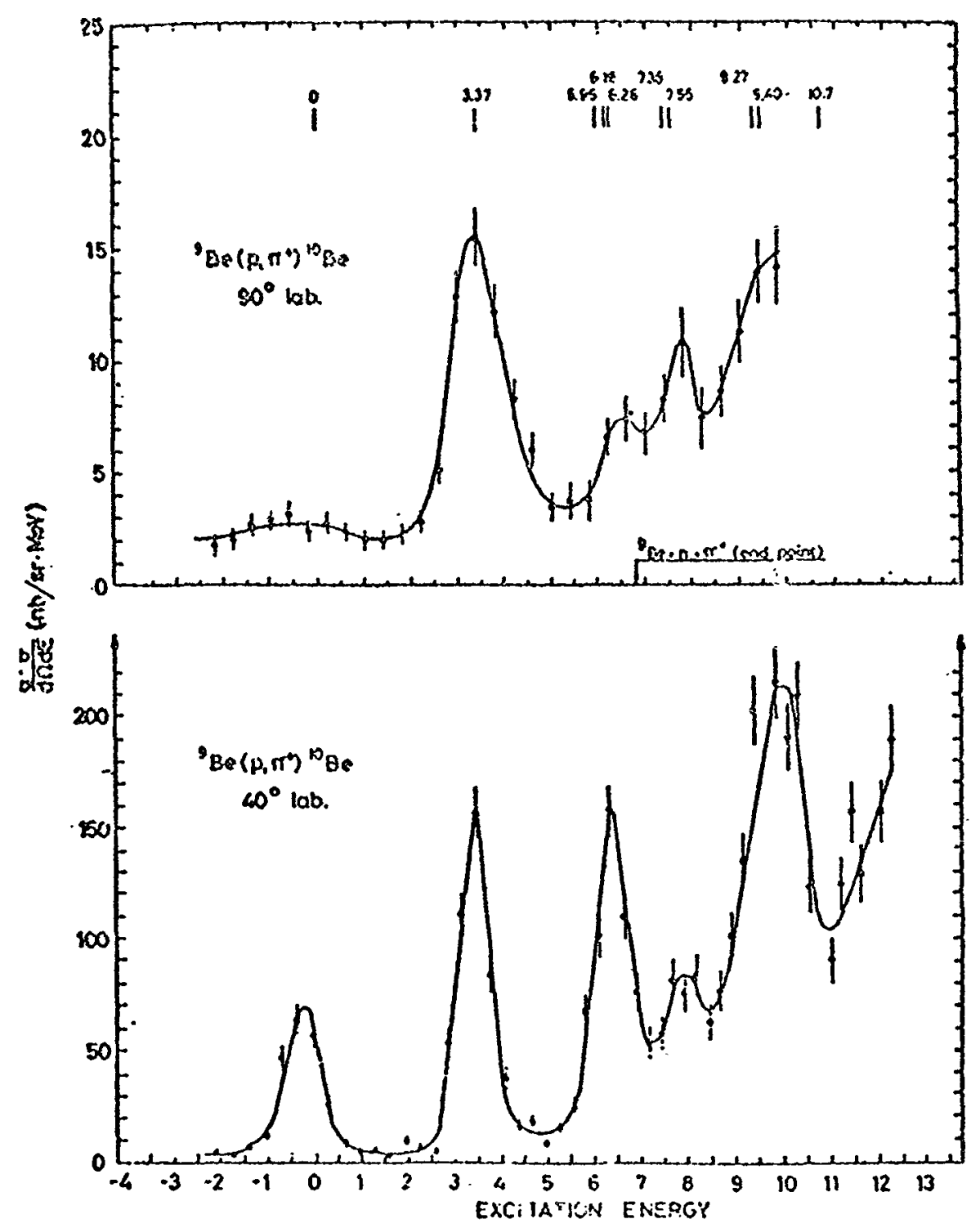

Fig. 10. - Energie d'excitation de la réaction ${ }^{11} \mathrm{Be}\left(\mathrm{p}, \Pi^{+}\right){ }^{10} \mathrm{Be}$ pour les angles de $90^{\circ}$ et $40^{\circ}$.

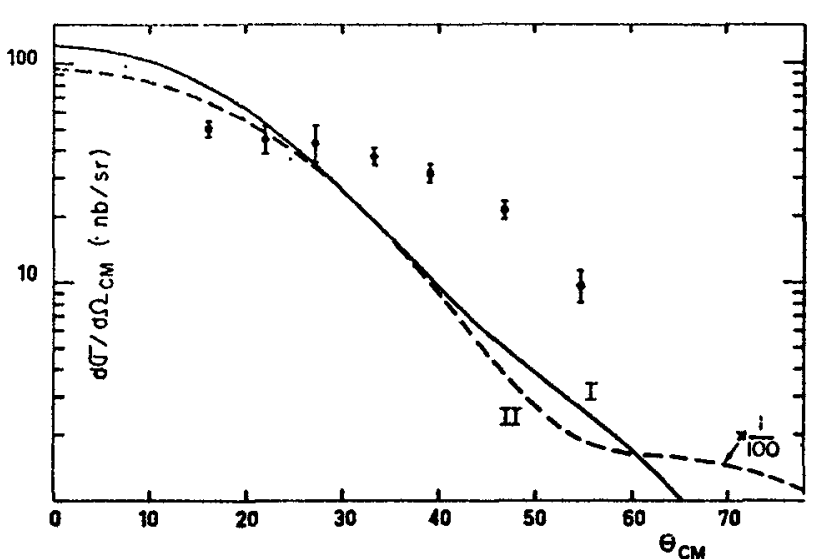

Fig. 11. - Distribution angulaire pour la réaction ${ }^{10} \mathrm{~B}\left(\mathrm{p}, \Pi^{+}\right)^{11} \mathrm{~B}_{\mathrm{gs}}$. Les courbes correspondent à deux calculs faits à partir d'une théorie à un nucléon.

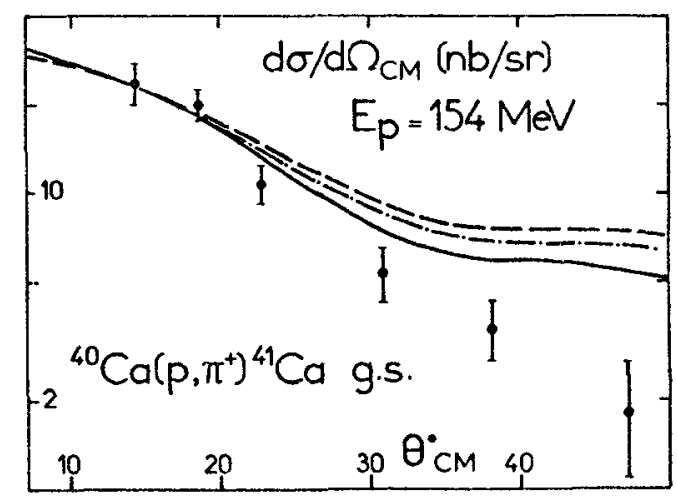

FIG. 12. - Distribution angulaire de la réaction ${ }^{40} \mathrm{Ca}\left(\mathrm{p}, \Pi^{+}\right)^{41} \mathrm{Ca}_{\mathrm{gs}}$ pour $T_{\mathrm{p}}=154 \mathrm{MeV}$. 


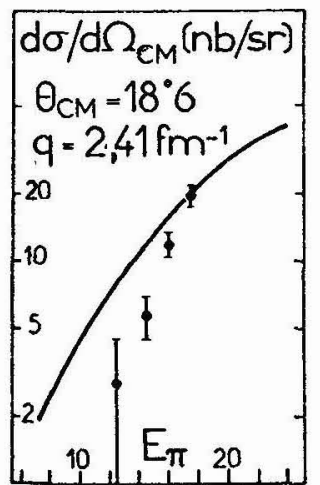

Fig. 13. - Section efficace à transfert constant $q=2,41 \mathrm{fm}^{-1}$ pour la réaction ${ }^{40} \mathrm{Ca}\left(\mathrm{p}, \Pi^{+}\right)^{41} \mathrm{Ca}$. La courbe correspond à un mécanisme à un nucléon.

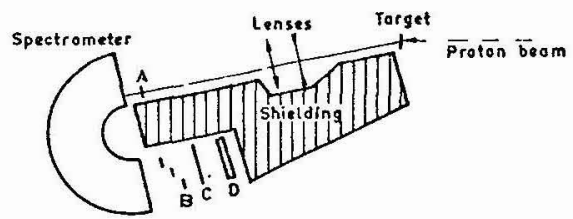

FIG. 14. - Dispositif expérimental utilisé par Le Bornec Y. et al.

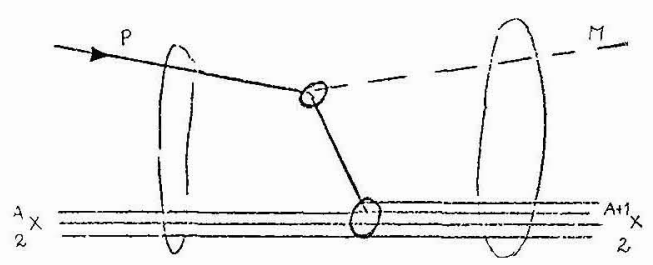

FIG. 15. - Mécanisme à un nucléon ${ }_{Z}^{A} \mathrm{X}\left(\mathrm{p}, \Pi^{+}\right)^{A+}{ }_{Z}^{1} \mathrm{X}$.

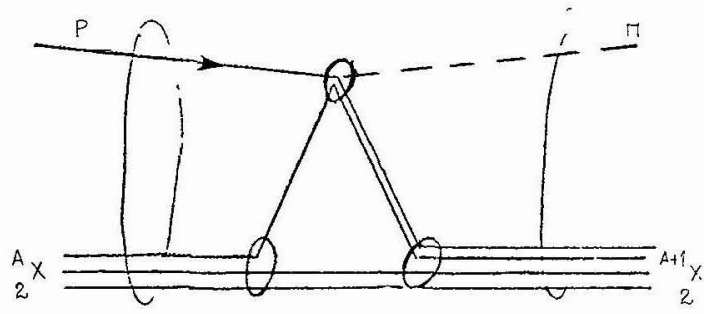

FIG. 16. - Mécanisme à deux nucléons ${ }_{2}^{A} \mathrm{X}\left(\mathrm{p}, \Pi^{+}\right)^{A+1}{ }_{2}^{\mathrm{X}}$.

Ces mesures reposent sur l'hypothèse que, puisque l'absorption est un processus à grand transfert d'énergie, l'approximation de l'impulsion est justifiée, et que le processus est quasi libre, le noyau résiduel restant spectateur dans une configuration à plusieurs trous, les nucléons correspondants emportant l'énergie du pion moins les énergies de liaison de ces nucléons. Partant de cette hypothèse, l'auteur étudie les noyaux résiduels en précisant leur niveau d'excitation par spectrométrie $\gamma$ fine (notons au passage que cela exclut toute étude de la production de l'état fondamental). Si le noyau excité a une durée de vie inférieure à $10^{-12} \mathrm{~s}$, l'auteur observe alors un élargissement Doppler de la raie $\gamma$ et compare celle-ci avec ce qu'il peut attendre d'un modèle :elatif à une distribution en moment de l'hypothétique cluster sur

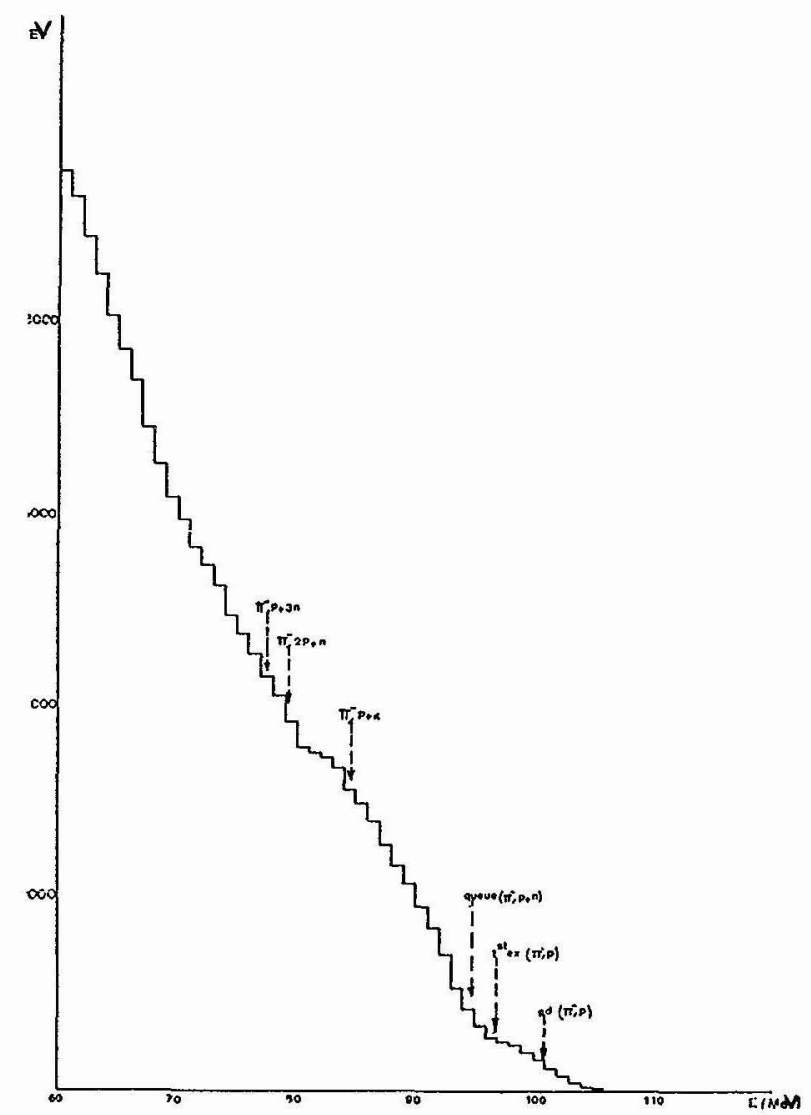

FIG. 17. - Spectre d'énergie des protons dans la réaction ${ }^{14} \mathrm{~N}\left(\Pi^{-}, \mathrm{p}\right){ }^{13} \mathrm{~B}$.

lequel l'absorption aurait lieu. A titre d'exemple, cette méthode appliquée à la réaction ${ }^{16} \mathrm{O}\left(\Pi^{-}, \mathrm{X}\right)^{12} \mathrm{C}^{*}$, le carbone 12 étant dans l'état $2^{+}$à $4,44 \mathrm{MeV}$ (Fig. 18), donne un élargissement compatible avec la distribution en moment, en supposant que $\mathrm{X}$ est un cluster $\alpha$, la distribution en moment ayant une valeur la plus probable de l'ordre de $200 \mathrm{MeV} / \mathrm{c}$.

Cet auteur donne aussi le rendement isotopique (Fig. 19) en fonction du nombre de nucléons enlevés

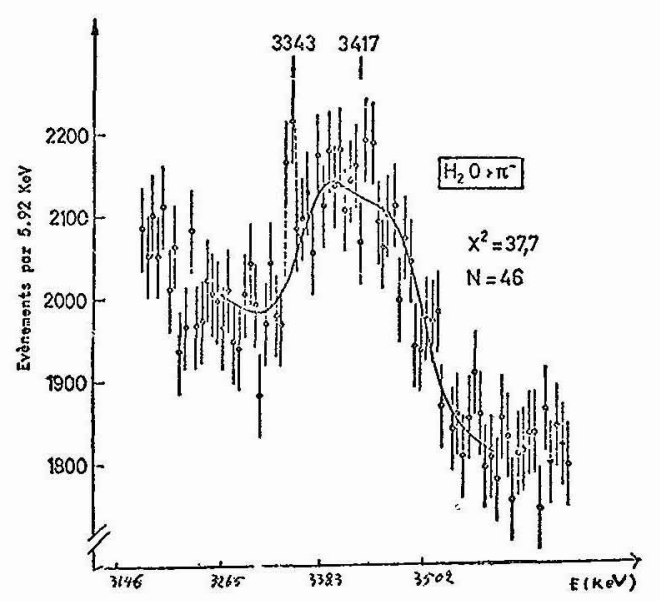

Fig. 18. - Raie $\gamma$ correspondant à la transition $2^{+} \rightarrow 0^{+} d u^{12} \mathrm{C}$ produit par l'absorption de $\Pi^{-}$sur ${ }^{16} \mathrm{O}$. La courbe continue représente le meilleur fit par un modèle de cluster. 


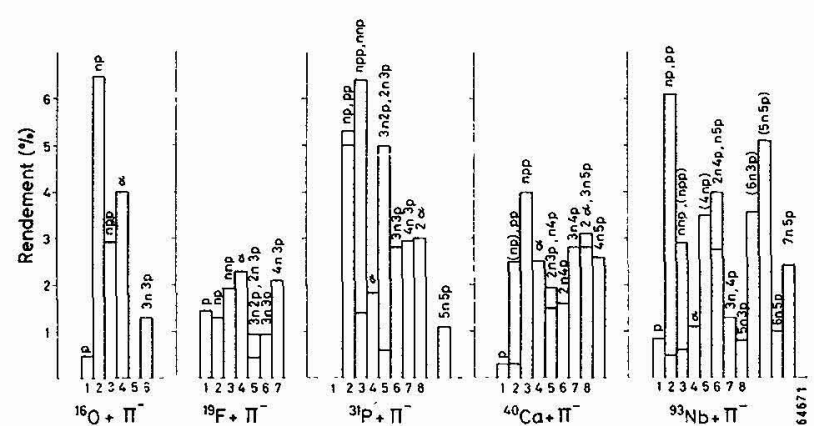

FIG. 19. - Rendement isotopique en fonction du nombre de nucléons enlevés au noyau cible.

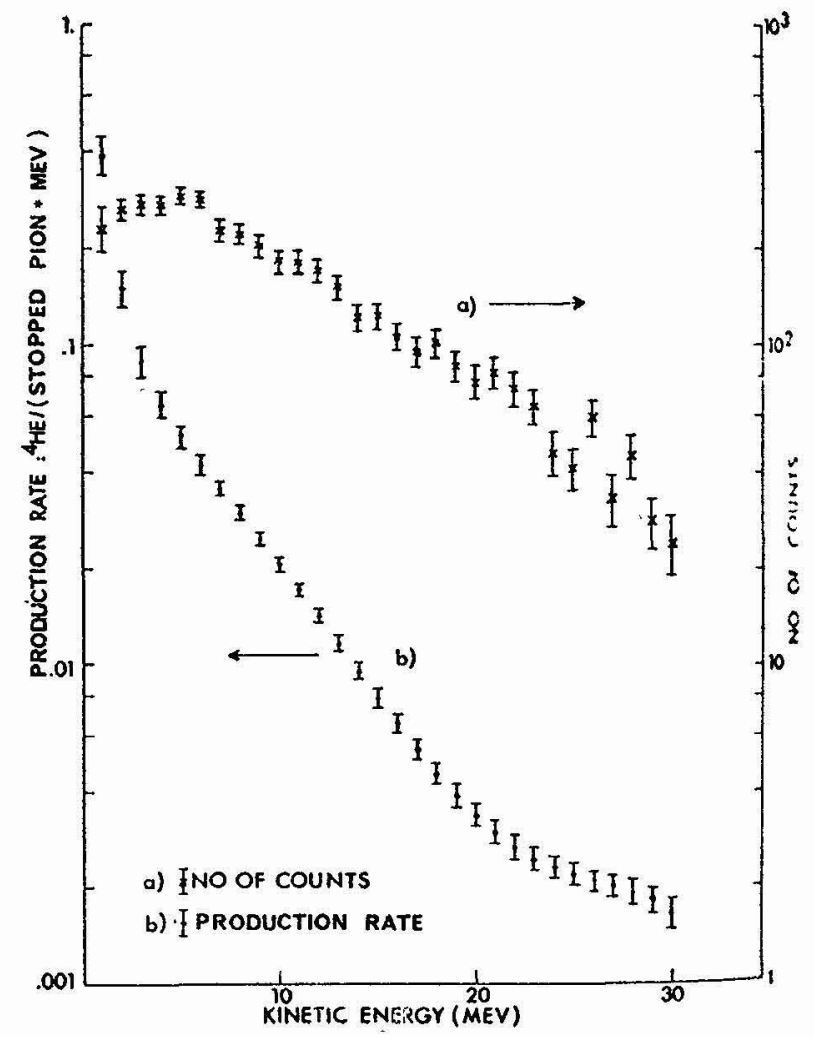

Fig. 20. - Taux de production des particules $\alpha$ en fonction de $E_{\alpha}$ pour la réaction ${ }^{12} \mathrm{C}(\Pi, \alpha) \mathrm{X}$.

à la cible pour des noyaux allant de ${ }^{16} \mathrm{O}$ à ${ }^{93} \mathrm{Nb}$. Il est à noter que la largeur de la distribution augmente avec $A$, ce qui a priori n'est pas choquant, l'hypothèse d'un processus quasi libre devenant de plus en plus faible.

Plus intéressante est l'inversion du rapport d'absorption sur les paires de nucléons np/pp qui a lieu autour de $A=31$, mais l'auteur ne peut que constater ce fait, et n'a aucune explication. De même, il est intéressant de voir que l'on enlève à la cible un nombre important de (2n, $2 p=\alpha)$ alors que d'autres combinaisons $(3 p, n)$, n'apparaissent pas, cela joue en faveur de cluster $\alpha$ dans le noyau. Citons en faveur de cette thèse les travaux récents de K. O. H. Ziock et al. [17] qui ont détecté les $\alpha$ suivant l'absorption de $\Pi^{-}$dans le ${ }^{12} \mathrm{C}$ (Fig. 15). Le nombre de particules $\alpha$ trouvé est grand $\left(1 \propto / \Pi^{-}\right.$stoppé).

4. Conclusion. - Ceci termine l'exposé des différentes communications que $\mathrm{j}$ 'avais à vous présenter. J'espère n'avoir pas pris trop de liberté avec les textes qui m'étaient proposés. Il me faut maintenant tirer les conclusions de ce que nous venons de voir.

Tout d'abord, le phénomène que l'on veut étudier est toujours plus complexe que nous l'avions espéré au début.

En effet, que ce soit :

- les expériences de D. Bachelier et al., où le mécanisme à un nucléon de type Pick-up n'a pas l'air de refléter les faits expérimentaux,

- les expériences de production de pion, où les calculs effectués ou en cours montrent qu'il faut envisager au moins des mécanismes à plusieurs nucléons, pour obtenir au moins les ordres de grandeur des sections efficaces

tout concourt à nous montrer que les nucléons dans le noyau ne peuvent pas être considérés comme indépendants, et que si l'on veut toucher l'un d'entre eux, au moins un autre est impliqué. Cela se fait-il par le moyen de corrélations à courte portée, ou par un mécanisme plus simple, qui aurait l'avantage de ne pas faire apparaître le mot corrélation qui montre notre ignorance? La question est ouverte.

Quoi qu'il en soit, ce domaine de la physique est excitant à étudier, et nous devons faire de nouvelles expériences plus complètes et plus précises.

Il est nécessaire de mesurer tous les paramètres de la réaction. Nous pouvons par exemple proposer de faire des expériences où l'on détecterait les particules émises, et dans le même temps, on connaîtrait l'état du noyau résiduel par une méthode analogue à celle utilisée par Engelhardt. Nous pouvons aussi envisager l'emploi d'appareillage mis au point par nos collègues des particules élémentaires, par exemple la chambre à streamer, où nous observerions tout le vertex chargé et où l'énergie des neutres serait mesurée par des techniques de temps de vol qui nous sont habituelles.

Si nous reprenons par exemple la figure 8 , il faut que nous repoussions la limite des seuils des réactions telles que $\left(\Pi^{+}, 2 \mathrm{p}\right)$ qui nous masquent les informations en dessus de celle-ci, c'est-à-dire reconnaître ce qui, en dessus de cette énergie, correspond à $\left(\Pi^{+}, \mathrm{p}\right)$ et $\left(\Pi^{+}, 2 p\right)$.

Il est aussi à noter que, puisqu'il y a une certaine évidence pour la présence de clusters dans les noyaux, et qu'une partie de l'absorption se fait par ceux-ci, il nous faut des mesures complètes et précises du phénomène d'absorption sur les noyaux légers ${ }^{3} \mathrm{He}$, ${ }^{+} \mathrm{He}, \mathrm{Li}$... 


\section{Bibliographie}

[1] BeRtin, P. Y. et al., Low energy $\Pi^{+}$p scattering. Sixth International Conference on High Energy Physics and Nucleon Structure, Santa $\mathrm{Fe}$ and Los Alamos, June 1975.

[2] Preedom, B. M. et al., An experimental study of the $\Pi^{+} \mathrm{d} \rightarrow \mathrm{pp}$ reaction at pion energies of 40,50 and $60 \mathrm{MeV}$. Sixth International Conference on High Energy Physics and Nucleon Structure, Santa Fe and Los Alamos, June 1975.

[3] Амato, J. et al., Meson Nucleus Interaction, Proceedings of International Seminar, Strasbourg septembre 1971.

[4] Witten, T. R. et al., Phys. Rev. 174 (1968) 1166.

[5] BACHelier, D. et al., Nucl. Phys. A 126 (1969) 60.

[6] Geruic, E. et al., Phys. Lett. 52B (1974) 39.

[7] DaHlgren, S. et al., Phys. Lett. 35B (1971) 219.

[8] Dahlgren, S. et al., Nucl. Phys. A 204 (1973) 53.

[9] Le BorneC, Y. et al., Phys. Lett. 49B (1974) 434.

[10] LE BorNEC, Y., Private Communication.
[11] Grossman, Z., Lenz, F., Locher, M. P., Ann. Phys. 84 (1974) 348.

[12] Delling, M., Hofman, H. M., Hubert, M. G., Phys. Lett. 44B (1973) 484.

[13] Coupat, B. et al., Phys. Lett. 55B (1975) 286.

[14] Coupat, B. et al., Sixth International Conference on High Energy Physics and Nucleon Structure, Santa Fe and Los Alamos, June 1975.

[15] EngelhardT, H. D., Thèse ès Sciences, Grenoble 1974.

[16] ENGELHARDT, H. D., Nucleon reaction induced by stopped Negative Pion, Symposium on Mesonic Effects in Nuclear Structure, Bonn 1974.

[17] ZıOCK, K. O. H. et al., $\alpha$ particle emission following $\Pi^{-}$capture in ${ }^{12} \mathrm{C}$, Sixth International Conference on High Energy Physics and Nuclear Structure, Santa Fe and Los Alamos, June 1975. 